\title{
The Radiology Department during the COVID-19 pandemic: a challenging, radical change
}

\author{
Letterio S. Politi ${ }^{1,2,3} \cdot$ Luca Balzarini $^{4}$ \\ Received: 31 March 2020 / Revised: 3 April 2020 / Accepted: 6 April 2020 / Published online: 21 April 2020 \\ (C) European Society of Radiology 2020
}

Dear Editor,

Since the first cases of SARS-CoV-2 pneumonia reported in Wuhan in December 2019 [1], the Coronavirus Disease 2019 (COVID-19) has rapidly spread worldwide, and Radiology Departments have to face significant changes in order to cope with this sanitary emergency [2]. We would like to share our experience with the outbreak of COVID-19 in our Radiology Department at the Humanitas Clinical and Research University Hospital, a tertiary level center located in Milan, Italy. In particular, we would like to share some actions we undertook in our activities that other Radiology Departments might ponder to take into consideration.

Since February 20, when an Italian 38-year-old man who had no travel history or prior contacts with people traveling from China was diagnosed positive for SARS-CoV-2 infection, Italy has registered 11,591 deaths over 101,739 positive cases by March the 30th, most of them concentrated in Lombardy (6818 deaths over 42,161 positive subjects), where Milan is located [3]. A few days after the first deadly cases, Italy started deploying tight security measures, including general lockdowns and social distancing [4] to try to flatten the epidemic curve and allow the healthcare system to be able to cope with the emergency. In this dramatic scenario, our

Letterio S. Politi

letterio.politi@hunimed.eu

1 Department of Neuroradiology, IRCCS Istituto Clinico Humanitas and Humanitas University, Via Alessandro Manzoni 56, 20089 Rozzano, MI, Italy

2 Department of Radiology and Hematology \& Oncology Division, Boston Children's Hospital, 300 Longwood Avenue, Boston, MA 02115, USA

3 Department of Radiology and Advanced MRI Center, University of Massachusetts Medical School and Medical Center, 55 Lake Avenue N, Worcester, MA 01655, USA

4 Department of Radiology, IRCCS Istituto Clinico Humanitas, Via Alessandro Manzoni 56, 20089 Rozzano, MI, Italy
Radiology Department, as well as others in the same area, underwent some important changes in the organization of daily activities to optimize the health care of both COVID-19 and non-COVID-19 patients. Here we aim to briefly describe the modifications and safety measures taken in our Radiology Department in order to face this sanitary emergency, ranging from optimal vetting, and setup of dedicated spaces, to optimization of the workflow and staff management. In our Institution, during the last 14 days, the number of hospitalized Covid-19 patients increased from 133 (18 of which in the intensive care units-ICUs) to 269 (41 of which in the ICUs), and every day a mean of 30 patients are diagnosed as positive for COVID-19 in our Emergency Department (ED).

Shortly after the state of emergency, a multidisciplinary team led by the Chief Medical Officer revised institutional COVID-19-control guidelines to guarantee the safety of both operators and patients during radiological exams and procedures.

1. In order to prevent the spreading of the disease within the hospital, separate ED, wards, and intensive care units (ICUs) were created for COVID-19 positive (or suspected positive) and non-COVID-19 patients, with dedicated medical and paramedical staff. The CT machine located within the original ED was dedicated exclusively to COVID-19 patients. Portable X-rays and ultrasound scanners were provided in each COVID-19 ICU and ward and were dedicated exclusively to these patients. The non-COVID-19 patients admitted in the ED, including acute stroke patients, had a separate pathway, and were studied with "clean" X-ray and CT scanners within the Radiology Department, previously dedicated to inpatients and outpatients. This helped to reduce the risk of in-hospital transmission and allowed to avoid time- and resource-consuming room decontamination procedures.

2. The radiographer staff rotating in the Emergency Radiology, handling COVID-19 positive patients, was 
doubled, and the time of each shift was reduced. This modification in the rotation was possible because all non-urgent and non-emergent activity within the hospital was postponed, except for oncology-related activities.

3. A triage for all outpatients and staff was set at the entrance of the hospital. A second triage was performed at the entrance of the Radiology Department. Systematic checks of temperatures were taken using hospital-issued digital thermometers. History of fever, cough, and contact with COVID-19 positive subjects was also evaluated.

4. Surgical masks were provided for all patients and staff at the entrance of the hospital and had to be worn for all the time.

5. Despite this double-triage procedure, a mean of 2.2 outpatients presenting lung CT findings typical for COVID19 pneumonia was found every day in the non-COVID pathway during the last 2 weeks. These were nonfebrile patients without previous history of contact with infected subjects, who underwent CT either for cough (typically lasting more than 2 weeks) or for cancer staging. For this reason, an accelerated protocol for room decontamination was set up, allowing the employment of the imaging room after just $30 \mathrm{~min}$ from positive case presence.

6. The number of outpatient radiological exams was drastically reduced to free resources for the COVID-19 patients and to allow enough distance among patients in the waiting rooms.

7. The participation to medical congresses and all inhospital meetings were banned. Participation to multidisciplinary boards was allowed for a single professional only for each discipline; a web-based videoconference service was established to allow participation of the whole board. Step-by-step instructions were provided to all operators to facilitate the videoconference connections.

8. All the healthcare professionals in the Radiology Department received specific training regarding safety measures through both an Institutional e-learning platform and dedicated training sessions in small groups. Counseling and psychological services were also established by the Hospital to assist staff exposed to risk of infection.

9. All the personal protective equipment (PPE) was available when facing a suspicious or confirmed COVID-19 patient. In case of interventional radiological or neuroradiological procedures, the following PPEs are always available: double sterile gloves, Ffp $2 / 3$ masks, nonsterile waterproof gown, and sterile surgical gown, adhering mask for eye protection.

10. For radiological intervention, two pathways were organized. First, an operating room equipped with C-arm, with a negative pressure environment, was set up in an isolated COVID-19 area to perform surgical, interventional radiology and hemodynamics procedures in COVID-19 patients. Second, non-COVID patients underwent interventional radiological procedures, in the "clean" angiographic suite in the Radiology Department. For urgent endovascular thrombectomy in either COVID-19 patients or suspected COVID-19 patients presenting acute stroke, the dedicated biplane neuro-angiographic suite was employed and decontaminated immediately after each procedure.

11. No observers or students were allowed to enter the Department. In addition to the checkpoints at the entrances of the hospital and of the Radiology Department, where dedicated personnel avoided the entrance of non-authorized people, signs were hung on the doors to remember the mandatory use of surgical masks and to prevent unauthorized staff from gaining access to the area. Residents' working schedule was temporarily halved.

In our opinion, the high collaboration among radiologists, radiographers, nurses, and other caregivers is critical to achieve the required safety measures within the Radiology Department and the whole hospital. Radiology must be ready to play an active role in the COVID-19 pandemic outbreak. This outbreak is both a sanitary emergency and an economic challenge for the whole healthcare system and also for Radiology Departments, facing a dramatic increase in the expenses and decrease in the revenue. However, it is our duty and mission to harmonize the services of the Radiology Departments with the compelling needs of the hospitals, and to contribute in the successful management of COVID-19 patients. We really hope that the dramatic situation that this part of Italy is now experiencing might not be faced in other countries.

Funding information The authors state that this work has not received any funding.

\section{Compliance with ethical standards}

Guarantor The scientific guarantor of this publication is Letterio S. Politi.

Conflict of interest The authors of this manuscript declare no relationships with any companies whose products or services may be related to the subject matter of the article.

Statistics and biometry No complex statistical methods were necessary for this Letter to the Editor.

Informed consent Written informed consent was not required for this study because this work is a Letter to the Editor. 
Ethical approval Institutional Review Board approval was not required because this work is a Letter to the Editor.

Methodology Not applicable.

\section{References}

1. Guan WJ, Ni ZY, Hu Y et al (2020) Clinical characteristics of coronavirus disease 2019 in China. N Engl J Med, https://doi.org/10. 1056/NEJMoa2002032
2. Mossa-Basha M, Meltzer CC , Kim DC, Tuite MJ, Kolli KP, Tan BS (2020) Department preparedness for Covid-19: radiology scientific expert panel. Radiology, https://doi.org/10.1148/radiol.2020200988

3. Protezione Civile Italiana. Available via http://opendatadpc.maps. arcgis.com/apps/opsdashboard/index.html\#/b0c68bce 2cce 478eaac82fe38d4138b1. Accessed 30 Mar 2020

4. China May Be Beating the Coronavirus, at a Painful Cost - The New York Times. Available via https://www.nytimes.com/2020/03/07/ world/asia/china-coronavirus-cost.html. Accessed 16 Mar 2020

Publisher's note Springer Nature remains neutral with regard to jurisdictional claims in published maps and institutional affiliations. 